\title{
Editorial
}

\section{La Revue canadienne du vieillissement entame sa dixième année}

La Reuue canadienne du vieillissement/Canadian Journal on Aging a connu un développement spectaculaire depuis la publication de son premier numéro en 1981. En diffusant largement les résultats de la recherche gérontologique, la Revue s'est montré un reflet du dynamisme de la communauté de la gérontologie canadienne, mais elle est aussi et surtout une participante active dans ce développement.

Avec la publication de ce numéro, la Revue entame sa dixième année. Ce moment est l'occasion de souligner que les deux rédacteurs en chef qui m'ont précédé, soit Mme Blossom Wigdor et M. Victor Marshall, ont accompli une oeuvre de pionniers et une oeuvre de géants. La gérontologie canadienne leur doit d'avoir fondé, maintenu et assuré l'avenir de La Revue canadienne du vieillissement, un véhicule de communication vitale entre chercheurs et entre chercheurs et praticiens. Ma tâche sera de continuer la leur, de poursuivre sur leur lancée. Aussi, la Revue ne subira pas de modifications substantielles dans les années à venir. Elle doit demeurer une revue savante avec les exigences de rigueur et de qualité que cela comporte.

La Revue canadienne du vieillissement est une revue savante. Elle vise à diffuser à un public de chercheurs, de praticiens et de personnes qui s'intéressent aux derniers développements dans le domaine de la gérontologie des connaissances de pointe. La Revue n'a jamais défini de façon étroite son domaine d'activités. Elle est une revue savante multidisciplinaire avec ses sections de biologie, de sciences de la santé, de psychologie, de sciences sociales et de pratique sociale. La large part des articles publiés dans la $R e$ vue sont scientifiques. À l'occasion, la Revue a publié des articles de réflexion, de philosophie ou d'analyse littéraire qui participaient tout aussi bien que des articles scientifiques stricto sensu au développement des connaissances en gérontologie. Ces articles avaient été soumis au processus d'arbitrage. Le comité de rédaction de la Revue veut maintenir cette large perspective multidisciplinaire dans la tradition d'une revue savante.

La connaissance ne se développe pas sans débat. Mais l'expression des désaccords est souvent limitée par les règles mêmes de l'arbitrage des articles. Si le processus d'arbitrage est essentiel pour garantir la qualité et la probité d'une revue scientifique, il peut aussi retarder les progrès. Ainsi, de nouvelles idées peuvent avoir de grandes difficultés à être diffusées malgré leur mérite. Pour éviter que cela se produise, sur suggestion d'un rédacteur de section, et après accord entre ce rédacteur et le rédacteur en chef, un article qui aura suscité un débat de fond qu'il est bon de rendre public, pourra paraître dans la Revue, dans la mesure où il sera accompagné d'une critique qui met en évidence l'objet même du débat et ses considérants. L'ar- 
ticle et son commentaire seront publiés simultanément dans une rubrique spéciale dite "Controverses". Par définition, peu d'articles seront publiés dans cette rubrique. Qui plus est, pour l'instant, nous ouvrons cette section "Controverses" à titre expérimental seulement. Le comité de rédaction de la Revue aimerait recevoir l'opinion de ses lecteurs sur cette rubrique.

Toujours pour animer le débat savant, la Revue accueillera favorablement des commentaires sur des articles déjà publiés. Ces commentaires devraient permettre l'échange de points de vue éclairés entre chercheurs autour de questions d'intérêt scientifique.

Enfin, la Revue est destinée à un audience qui dépasse largement les frontières de la communauté des chercheurs en gérontologie. La Revue est un pivot de communication entre chercheurs et praticiens de la gérontologie. Mais les articles scientifiques sont souvent arides par définition, tandis que La Revue canadienne du vieillissement n'est pas une revue de vulgarisation scientifique. Il y a cependant lieu d'accompagner des articles scientifiques d'opinions éclairées sur la signification pour la pratique des travaux de recherche. Certains résultats de recherche se prêtent mieux que d'autres à cet exercice. Ici encore, nous entendons solliciter l'expression d'opinion avec parcimonie. Le but n'est pas ici de provoquer la controverse mais de susciter la réflexion, d'éveiller aux conséquences sociales, éthiques et philosophiques des résultats de recherche. Ces textes seront courts, sollicités par le comité de rédaction de la Revue. Ils seront publiés dans la rubrique "Editorial".

En somme, nous maintenons que l'objet principal de la Revue est la publication de textes savants, la communication de connaissances à un auditoire de chercheurs et de praticiens de la gérontologie. Essentiellement, la Revue demeurera donc ce qu'elle est et le processus d'arbitrage des textes s'appliquera strictement. La Revue s'efforcera cependant de réfléter plus adéquatement l'activité savante réelle. Occulter le débat revient à couvrir l'activité savante du voile pudique de l'unanimité qui lui nuit. Mais ce débat doit lui aussi être encadré par des règles. Dans le cas qui nous préocupe, le comité de rédaction s'est donné, à titre expérimental, un petit nombre de règles qui ont été exposées ci-haut. Nous en tenterons l'expérience et nous nous donnons le privilège de la terminer, si nécessaire, à notre convenance. La Revue désire aussi tenir compte des intérêts d'une grande partie de lecteurs qui y cherchent de l'inspiration pour leur pratique. L'éditorial poursuit ce but.

Enfin, une revue savante se fait essentiellement grâce aux auteurs. Je veux ici exprimé tout mon respect aux chercheurs qui soumettent des manuscrits à la Revue. Je veux les assurer de mon plus grand intérêt au maintien de la haute qualité scientifique de la Revue. Elle cherche à mettre en évidence les textes qui sont publiés, à permettre aux idées de circuler le plus librement possible dans la communauté scientifique et professionnelle, à l'intérieur de règles rigoureuses qu'il est de mise de respecter pour garantir une revue de la meilleure qualité possible.

François Béland, Rédacteur en chef 